\title{
Diferencias clínicas en pacientes con apnea obstructiva del sueño de carácter postural
}

\author{
JORGE JORQUERA A.*, GONZALO LABARCA T.**, JORGE DREYSE D.*** y CONSTANZA SALAS C.*
}

\section{Clinical differences in positional obstructive sleep apnea patients}

Introduction: Obstructive sleep apnea syndrome (OSA) is the result of repetitive collapse of the upper airway during the sleep. There is a group of patients with a positional component; which is defined as a reduction of at least 50\% in the apnea hypopnea index (AHI) from the supine to a lateral position. Objective: The purpose of this study is to determine positional OSA prevalence and compare clinical characteristics and polygraph recordings among this group of patients. Methods: Prospective study of polygraph recording of patients with suspect of OSA between 2009-2014. Demographic, anthropometric, comorbidities and polygraph recording data was collected. OSA was defined as AHI $>5$ events per hour. Results: From a total of 457 patients with OSA diagnose, 243 had a positional component (53\%). Positional Sleep Apnea patients had mild AHI $(p<0.001)$, a lower neck circumference $(p=0.001)$, lower Body Mass Index $(p=0.003)$ and a lower presence of arterial hypertension $(p=0.001)$. The multivariable analysis confirmed that there is a lower probability in Positional OSA in patients with Severe AHI (OR 0,96: IC 0,94-0,97), with hypertension (0,54: IC 0,33-0,88) and males (OR 0,41: IC 0,21-0,81). Conclusions: Positional obstructive sleep apnea is a very frequent condition presented in $53 \%$ of OSA patients; these patients have often mild AHI, lower BMI, lower neck circumference, less hypertensive, and are usually females.

Key words: Sleep apnea syndrome; Sleep apnea, obstructive; Prospective studies; comorbidity; Positional sleep apnea, Body posture, sleep position.

\section{Resumen}

Introducción: El síndrome de apnea e hipopnea obstructiva del sueño (SAHOS) es producto de un colapso repetido a nivel de la vía aérea superior, durante el sueño. Existe un grupo de pacientes con SAHOS con componente postural, definida por una reducción del 50\% o más en el índice de apnea hipopnea (IAH) de la posición supina a la lateral. Objetivo: Determinar la prevalencia de SAHOS postural y comparar las características clínicas y de la poligrafia respiratoria $(P R)$ de este grupo de pacientes. Metodología: Estudio prospectivo de PR entre 2009-2014, derivados a realización de estudio por sospecha clínica de SAHOS. Se recolectaron datos demográficos, antropométricos, comorbilidades $y$ diferentes variables de la PR. Se definió presencia de SAHOS un IAH mayor de 5 eventos $/$. Resultados: De un total de 457 pacientes con diagnóstico de SAHOS, 243 presentaban SAHOS postural (53\%). Los pacientes con SAHOS postural eran más leves $(p<0,001)$, tenían una circunferencia cervical $(p=0,001)$ e indice de masa corporal $(p=0,003)$ menor y con menor frecuencia eran hipertensos $(p=0,001)$. El análisis multivariado confirmó menor probabilidad SAHOS postural en sujetos con IAH graves (OR 0,96: IC 0,94-0,97): hipertensos (0,54: IC 0,33-0,88) y de sexo masculino (OR 0,41: IC 0,21-0,81). Conclusiones: El SAHOS postural es una entidad muy frecuente que está presente en el 53\% de los pacientes con SAHOS, estos sujetos tienden a presentar enfermedad más leve, tener un IMC y circunferencia cervical menor, menor frecuencia de hipertensión arterial y son preferentemente mujeres.

Palabras clave: Síndrome Apnea del sueño; Apnea del sueño obstructiva; Estudios prospectivos; comorbilidad; apnea postural, apnea posicional.

\footnotetext{
* Centro de Enfermedades Respiratorias, Departamento de Medicina Interna. Clínica Las Condes. Región Metropolitana, Chile.

** Facultad de Medicina, Universidad San Sebastián. Concepción y Departamento de Medicina Interna. Complejo Asistencial Víctor Ríos Ruiz. Los Ángeles, Chile.

***Centro de Pacientes Críticos, Clínica Las Condes. Región Metropolitana, Chile.
} 


\section{Introducción}

El síndrome de apnea e hipopnea obstructiva del sueño (SAHOS), es una enfermedad frecuente que afecta al $4 \%$ de la población adulta ${ }^{1,2}$, produce como síntoma cardinal somnolencia diurna excesiva ${ }^{3}$, y se ha asociado a mayor riesgo de hipertensión arterial ${ }^{4-7}$, accidentes de tránsito $^{8-9}$, laborales y mayor morbimortalidad cardiovascular $^{10-12}$. Los eventos de apnea e hipopnea son producto de un colapso total o parcial de la vía aérea superior. La posición supina juega un rol importante en estos eventos ya que la fuerza de gravedad promueve el movimiento hacia atrás de la base de la lengua y las partes blandas, mientras que este desplazamiento ocurre en menor grado en decúbito lateral. Existe un grupo de pacientes con SAHOS con componente postural, la cual está definida por una reducción del $50 \%$ o más en el índice de apnea e hipoapnea de la posición supina a la lateral y estaría presente entre el 50 al $60 \%$ de los individuos con SAHOS ${ }^{13-16}$. Si bien el tratamiento de elección en los pacientes con enfermedad grave y sintomática es la ventilación con presión positiva continua de la vía aérea a través de una máscara nasal (CPAPn) ${ }^{17-19}$, el grupo de pacientes con SAHOS postural podría verse beneficiado por medidas posicionales ${ }^{20}$. Sin embargo, la terapia posicional podría no aliviar los síntomas si el índice en decúbito lateral permanece elevado, por lo que la definición clínica más apropiada debería exigir además que el índice de eventos respiratorios en decúbito lateral sea menor de un umbral clínico adecuado. La prevalencia del SAHOS postural en estudios de poligrafía respiratoria no se conoce como tampoco la prevalencia de esta definición más estricta, lo cual tiene implicancias terapéuticas relevantes. Este estudio está diseñado para evaluar la prevalencia de apneas posturales y determinar las características clínicas y de la poligrafía respiratoria de estos pacientes.

\section{Pacientes y Métodos}

\section{Sujetos}

Se evaluó en forma prospectiva los pacientes adultos sometidos a estudio de sueño por la sospecha clínica de SAHOS mediante poligrafía respiratoria (PR) ambulatoria entre 2009-2014. Un total de 585 pacientes fueron ingresados para realizar el estudio. Antes de realizar el estudio diagnóstico, se aplicó una encuesta estandarizada de síntomas, con el propósito de evaluar el horario de sueño, grado de somnolencia diurna, ronquido, apneas presenciadas, insomnio, episodios de sofocación nocturna, nicturia, cefalea matinal, boca seca al despertar, pérdida de memoria y deterioro cognitivo. Se consignaron los datos sociodemográficos, hábitos, comorbilidades y los datos antropométricos, tales como peso, talla y circunferencia cervical, abdominal y de cintura, entre otras. De esta forma se recolectaron datos demográficos, antropométricos, comorbilidades y las diferentes variables de la PR; índice de apnea hipoapnea (IAH), saturación media y mínima de oxígeno, tiempo con saturación bajo $90 \%$, tiempo total de registro, porcentaje en diferentes posiciones, etc. A todos los pacientes se les aplicó una encuesta de síntomas que incluía entre otras la escala de somnolencia de Epworth ${ }^{21}$, escala de Thorton modificada, Stanford y escala de depresión de Beck $^{22}$. El estudio fue aprobado por el Comité de Ética de la institución y los pacientes firmaron el acta de consentimiento informado previo a su incorporación.

\section{Poligrafía respiratoria}

Para la realización de la poligrafía respiratoria (PR) se utilizó un equipo marca Embletta o Embletta gold (Natus sleep products, EE. UU.) que cumple con las exigencias de la Academia Americana de Medicina del Sueño (AASM) para los estudios de nivel $\mathrm{III}^{23}$, que incluía el registro de flujo nasal mediante cánula nasal, movimientos tóraco abdominales (xtrace RIP belts: pletismografía de inductancia), frecuencia cardiaca y saturación de oxígeno a través de un oxímetro de pulso digital y sensor de posición. Los estudios fueron realizados en el domicilio, previa instrucción en el de laboratorio de enfermedades respiratorias para la instalación de este a cargo del paciente o de su familia en su domicilio. El registro debía cumplir con los estándares exigidos AASM para estudios diagnósticos, en caso contrario era repetido. El análisis de la PR fue realizado manualmente por un único médico especialista en enfermedades respiratorias con amplia experiencia en el diagnóstico de trastornos respiratorios del sueño, de acuerdo a las normas de la $\mathrm{AASM}^{24}$ empleando las siguientes definiciones: Se definió apnea como la ausencia de flujo por más de $10 \mathrm{~s}$ : a) Apnea obstructiva: ausencia del flujo oronasal en presencia de movimientos tóraco abdominales; b) Apnea central: ausencia de flujo oronasal y de los movimientos tóraco abdominales; c) Apnea mixta: ausencia de flujo oronasal que comienza con un componente 
central y termina como obstructiva; y d) Hipoapneas: reducción del flujo aéreo discernible (más de 30\% y menos del $90 \%$ ) por un período superior a $10 \mathrm{~s}$. Todos los eventos respiratorios deben presentar una caída de la saturación de oxígeno de al menos 3\%. Se calculó el número de eventos respiratorios dividiendo el número total de eventos respiratorios por el tiempo total de registro en horas (número de apneas más número de hipoapneas dividido por tiempo total de registro). Los hallazgos fueron considerados diagnósticos de SAHOS cuando el número de eventos respiratorios fue mayor o igual a cinco eventos por hora. El SAHOS fue clasificado según gravedad en leve (5 a15 eventos/h); moderado (15 a 30 eventos $/ \mathrm{h})$ y grave $(>30$ eventos/h $)^{25}$. Fueron incluidos al estudio sólo aquellos exámenes cuyo registro presentaba al menos una hora de registro en decúbito dorsal y lateral. Se definió apnea postural (AP) si se observaba una reducción mayor un $50 \%$ del número de eventos respiratorios entre el decúbito supino y el decúbito lateral. Además, se clasificó los pacientes en AP estricta en la cual además de disminuir más del $50 \%$ de los eventos respiratorios en decúbito lateral estos presentaban menos de diez eventos por hora registro.

\section{Análisis estadístico}

Los resultados fueron expresados como valores promedio \pm desviación estándar para las variables medidas en escala numérica y en porcentaje para las medidas en escala nominal. Las variables cualitativas fueron comparadas mediante la prueba de $\chi^{2}$ y test exacto de Fisher, y las variables continuas con la prueba $t$ de Student. Las variables clínicas y de la poligrafía respiratoria relacionadas con el SAHOS fueron sometidas a análisis univariado y multivariado en un modelo de regresión logística (modalidad stepwise). Se calcularon los odds ratio (OR) e intervalos de confianza (IC) para el 95\%. En adición, se realizó un modelo de comparación múltiple mediante test de ANOVA con la finalidad de encontrar diferencias entre los distintos grupos de pacientes según resultado de su PR: pacientes con AP estricta: pacientes con AP no estricta (AP-AP estricta) y pacientes sin AP. Las variables incluidas en este análisis fueron edad, IAH, circunferencia de cuello, IMC y escala de Epworth. Los análisis y el registro de los datos se realizaron mediante el software Excell 2011 y el software SPSS 12.0 (SPSS Inc., Chicago). Se consideró una significancia estadística un valor de $\mathrm{p}<0,05$.

\section{Resultados}

Se evaluaron mediante poligrafía respiratoria, 585 sujetos adultos con sospecha clínica de SAHOS de los cuales 503 presentaban registro suficiente en ambas posiciones para poder definir la presencia de apneas posturales. El 91\% de los sujetos estudiados (457/503) presentaban diagnóstico de SAHOS. La edad promedio fue $52 \pm 13$ años (rango: 16-85), 80\% eran varones, $76 \%$ obesos (IMC $>25 \mathrm{~kg} / \mathrm{m}^{2}$ ), $28 \%$ fumadores activos y $72 \%$ tenían un IA/H mayor de 15 eventos/h. En la Tabla 1 se describen las características clínicas y de la poligrafía respiratoria de los pacientes con SAHOS incluidos en el estudio. De un total de 457 pacientes con diagnóstico de SAHOS, 243 presentaron diagnóstico de SAHOS postural (53\%) y 136 cumplieron criterio de AP estricta $(29,7 \%)$. Cuando se analizó por gravedad de la SAHOS, presentaban apneas posturales el 75\% (95/126) de los pacientes leves, el $62 \%$ $(79 / 128)$ de los pacientes moderados y el $34 \%$ $(69 / 203)$ de los graves. Las diferencias de los

Tabla 1. Características clínicas y de la poligrafía respiratoria de la población incluida en el estudio

\begin{tabular}{|c|c|}
\hline Edad, años ( \pm DS) & $52,27( \pm 13,23)$ \\
\hline Sexo masculino, $\mathrm{n}$ & $369(80 \%)$ \\
\hline Circunferencia cervical, cm ( \pm DS) & $39,2( \pm 14,28)$ \\
\hline Índice de masa corporal, $\mathrm{kg} / \mathrm{m}^{2}$ & $41,68( \pm 12,02)$ \\
\hline Hipertensión arterial, n & $235(51,6 \%)$ \\
\hline Diabetes Mellitus, n & $134(29,3 \%)$ \\
\hline Escala de Epworth ( \pm DS) & $10,2( \pm 0,7)$ \\
\hline $\begin{array}{l}\text { Variables poligrafía respiratoria } \\
\text { Ronquido, } \mathrm{n} \\
\text { Tiempo total registro (min) }( \pm \mathrm{DS}) \\
\text { IAH ( } \pm \text { DS) } \\
\text { Duración media apneas }(\mathrm{s})( \pm \mathrm{DS}) \\
\text { ID- } 4 \text { ( } \pm \text { DS) } \\
\text { Saturación de } \mathrm{O}_{2} \text { promedio }( \pm \mathrm{DS}) \\
\text { Saturación mínima de } \mathrm{O}_{2} \\
\text { CT-90\% }( \pm \mathrm{DS})\end{array}$ & $\begin{array}{l}297(64,9 \%) \\
483(76) \\
26,61( \pm 11,17) \\
20,7(5,6) \\
28(18,2) \\
92,3(3,1) \\
77,8(10,1) \\
18(24)\end{array}$ \\
\hline $\begin{array}{l}\text { Severidad } \\
\text { Leve } \\
\text { Moderado } \\
\text { Severo }\end{array}$ & $\begin{array}{l}126(27,5 \%) \\
128(28 \%) \\
203(44,4 \%)\end{array}$ \\
\hline $\begin{array}{l}\text { Clasificación tipo de AOS } \\
\text { AP postural } \\
\text { AP estricta } \\
\text { AP no estricta }\end{array}$ & $\begin{array}{l}243(53,1 \%) \\
136(29,8 \%) \\
107(23,4 \%)\end{array}$ \\
\hline Apnea no postural & $214(46,8 \%)$ \\
\hline
\end{tabular}

DS: Desviación standard; IAH: Índice Apnea hipoapnea; ID-4: Índice de desaturación > 4\%; CT-90\%: \% de tiempo con $\mathrm{SaO}_{2}<90 \%$; AP: Apnea postural. 
Tabla 2. Análisis uni y multivariado sobre las diferencias clínicas de pacientes con diagnóstico de Síndrome de Apnea Hipopnea Obstructiva del sueño -SAHOS- postural y no postural

\begin{tabular}{|c|c|c|c|c|c|}
\hline \multirow[b]{2}{*}{ Variable } & \multicolumn{2}{|c|}{ Análisis univariado } & \multirow[b]{2}{*}{ Valor de $p$} & \multicolumn{2}{|c|}{ Análisis multivariado } \\
\hline & $\begin{array}{l}\text { Postural } \\
(\mathrm{n}=243)\end{array}$ & $\begin{array}{l}\text { No postural } \\
(\mathrm{n}=214)\end{array}$ & & OR & IC \\
\hline Sexo masculino & 188 & 155 & 0,12 & 0,41 & $(0,21-0,81)^{*}$ \\
\hline Edad (media \pm DS) & $54,18(14,07)$ & $55,55(13,47)$ & 0,29 & 1,003 & $(0,98-1,02)$ \\
\hline IMC (media \pm DS) & $30,90 \quad(8,55)$ & $32,84 \quad(5,50)$ & 0,00 & 0,99 & $(0,96-1,02)$ \\
\hline Cuello (media \pm DS) & $41,60 \quad(4,28)$ & $43,01 \quad(4,15)$ & 0,00 & 0,94 & $(0,87-1,01)$ \\
\hline HTA & 103 & 132 & $<0,001^{*}$ & 0,54 & $(0,33-0,88)^{*}$ \\
\hline DM2 & 60 & 66 & 0,09 & 1,03 & $(0,61-1,72)$ \\
\hline Epworth (media \pm DS) & $10,33 \quad(5,24)$ & $10,91 \quad(5,90)$ & 0,27 & 1 & $(0,96-1,04)$ \\
\hline Ronquido & 155 & 126 & 0,43 & 0,96 & $(0,94-0,97)^{*}$ \\
\hline $\mathrm{IAH}($ media \pm DS) & $22,87(15,56)$ & $40,84(24,77)$ & $<0,001^{*}$ & 1,44 & $(0,09-2,40)$ \\
\hline
\end{tabular}

IMC: Índice de masa corporal; Cuello: perímetro cervical; HTA: hipertensión arterial. DM2: Diabetes mellitus 2. Epworth (escala de somnolencia diurna). DS: Desviación estándar, OR > Odds ratio. IC: Intervalo de confianza. *Se consideró un valor $\mathrm{p}<0,05$ como significativo. IAH: índice de apnea hipopnea.

Tabla 3. Análisis por comparaciones múltiples (ANOVA) entre los distintos grupos evaluados

\begin{tabular}{|c|c|c|c|c|c|}
\hline Variable & Grupo & Comparador & Diferencia media & Intervalo confianza & Valor $p$ \\
\hline Edad & $\begin{array}{l}1 \\
2 \\
3\end{array}$ & $\begin{array}{l}2 \\
3 \\
1 \\
3 \\
1 \\
2\end{array}$ & $\begin{array}{r}1,05 \\
-0,79 \\
-1,05 \\
-1,84 \\
0,79 \\
1,84\end{array}$ & $\begin{array}{ll}(-3,15 ; & 5,24) \\
(-4,63 ; & 3,05) \\
(-5,24 ; & 3,15) \\
(-5,39 ; & 1,72) \\
(-3,05 ; & 4,63) \\
(-1,72 ; & 5,39)\end{array}$ & $\begin{array}{l}0,83 \\
0,88 \\
0,83 \\
0,45 \\
0,88 \\
0,45\end{array}$ \\
\hline Índice masa corporal & $\begin{array}{l}1 \\
2 \\
3\end{array}$ & $\begin{array}{l}2 \\
3 \\
1 \\
3 \\
1 \\
2\end{array}$ & $\begin{array}{r}0,25 \\
-1,79 \\
-0,25 \\
-2,05 \\
1,79 \\
2,05\end{array}$ & $\begin{array}{rr}-1,87 ; & 2,37) \\
(-3,73 ; & 0,15) \\
(-2,37 ; & 1,87) \\
(-3,84 ; & -0,24) \\
-0,15 ; & 3,73) \\
(0,24 ; & 3,84)\end{array}$ & $\begin{array}{c}0,96 \\
0,08 \\
0,96 \\
0,02^{*} \\
0,08 \\
<0,01^{*}\end{array}$ \\
\hline Circunferencia cervical & $\begin{array}{l}1 \\
2 \\
3\end{array}$ & $\begin{array}{l}2 \\
3 \\
1 \\
3 \\
1 \\
2\end{array}$ & $\begin{array}{r}2,20 \\
-0,15 \\
-2,20 \\
-2,35 \\
0,15 \\
2,35\end{array}$ & $\begin{array}{rr}(0,92 ; & 3,48) \\
(-1,33 ; & 1,02) \\
(-3,48 ; & -0,92) \\
(-3,43 ; & -1,27) \\
(-1,02 ; & 1,33) \\
(1,27 ; & 3,43)\end{array}$ & $\begin{array}{c}<0,01^{*} \\
0,95 \\
<0,01^{*} \\
<0,01^{*} \\
0,95 \\
<0,01^{*}\end{array}$ \\
\hline Epworth & $\begin{array}{l}1 \\
2 \\
3\end{array}$ & $\begin{array}{l}2 \\
3 \\
1 \\
3 \\
1 \\
2\end{array}$ & $\begin{array}{r}0,84 \\
-0,11 \\
-0,84 \\
-0,95 \\
0,11 \\
0,95\end{array}$ & $\begin{array}{ll}(-0,85 ; & 2,53) \\
(-1,65 ; & 1,44) \\
(-2,53 ; & 0,85) \\
(-2,39 ; & 0,49) \\
(-1,44 ; & 1,65) \\
(-0,49 ; & 2,39)\end{array}$ & $\begin{array}{l}0,47 \\
0,99 \\
0,47 \\
0,27 \\
0,99 \\
0,27\end{array}$ \\
\hline Índice apnea hipopnea & $\begin{array}{l}1 \\
2\end{array}$ & $\begin{array}{l}2 \\
3 \\
1 \\
3 \\
1 \\
2\end{array}$ & $\begin{array}{r}18,96 \\
-7,37 \\
-18,96 \\
-26,33 \\
7,37 \\
26,33\end{array}$ & $\begin{array}{c}(13,11 ; 24,79) \\
(-12,72 ;-2,015) \\
(-24,79 ;-13,11) \\
(-31,28 ;-21,36) \\
(2,01 ; 12,72) \\
(21,36 ; 31,28)\end{array}$ & $\begin{array}{l}<0,01^{*} \\
<0,01^{*} \\
<0,01^{*} \\
<0,01^{*} \\
<0,01^{*} \\
<0,01^{*}\end{array}$ \\
\hline
\end{tabular}

Grupo 1: Apnea Postural (AP) estricta; Grupo 2: AP no estricta; Grupo 3: SAHOS no postural. *p $<0,05$. 
pacientes con SAHOS postural y no postural se muestran en la Tabla 2. En resumen, los pacientes con SAHOS postural eran menos graves, tenían una circunferencia cervical e índice de masa corporal (IMC) menor y con menor frecuencia eran hipertensos, el resto de las comorbilidades fueron similares en ambos grupos y no presentaban diferencia en edad, género ni grado de somnolencia medido mediante la escala de Epworth. Tampoco hubo diferencias en la escala de Thorton modificada, escala de somnolencia de Stanford ni en la escala de depresión de Beck. El análisis multivariado confirmó que existe menor probabilidad de presencia de apnea postural (AP) en sujetos con SAHOS severo (OR 0,96: IC 0,94-0,97): en pacientes hipertensos $(0,54$ : IC $0,33-0,88)$ y en pacientes del sexo masculino (OR 0,41: IC 0,21-0,81). Los pacientes con la definición más estricta de SAHOS postural: presentan una circunferencia cervical menor, sin embargo, no se encontraron diferencias en cuanto a la edad, IMC, escala de somnolencia de Epworth ni índice de eventos respiratorios en relación a SAHOS no postural. El resultado del análisis univariado y multivariado se muestra en la Tabla 2. El modelo de comparación múltiple analizando las diferencias entre los pacientes con AP estricta, AP no estricta y Apnea no Postural (A no P), evidencio diferencias significativas en la circunferencia cervical (CC) entre el grupo de AP estricta y no estricta, presentando una $\mathrm{CC}$ dos $\mathrm{cm}$ menor $(\mathrm{p}<0,01)$. También observamos diferencias significativas en relación al IAH entre todos los grupos analizados (Tabla 3).

\section{Discusión}

El SAHOS postural definido como la reducción del 50\% o más en el índice de eventos respiratorios al cambiar de la posición supina a la lateral es una entidad muy frecuente y se presenta en el 53\% de los pacientes con SAHOS. Este es el primer reporte que tenemos conocimiento de la prevalencia de SAHOS postural realizado con estudio de poligrafía respiratoria en domicilio y observamos una prevalencia similar a la descrita con estudios de polisomnografía (PSG) realizada en clínicas de sueño ${ }^{13-16}$. Además, la prevalencia es similar al reporte realizado por el primer autor de este estudio que encontró un $48 \%$ de prevalencia en los estudios de PSG en la clínica de la Pontificia Universidad Católica de Chile $^{26}$. Aun utilizando un criterio más estricto de $\mathrm{AP}(\mathrm{IAH}<10$ eventos/h en decúbito lateral) esta condición sigue siendo muy frecuente, siendo identificada en el $29,8 \%$ de los pacientes con SAHOS, similar a lo publicado por Mador et $\mathrm{al}^{14}$ donde se encontró una prevalencia de $27,5 \%$ pero con un nivel de corte en decúbito lateral de 5 eventos/h. El punto de corte a utilizar en decúbito lateral no está establecido, nosotros preferimos utilizar 10 eventos $/ \mathrm{h}$ en vez de 5 eventos/h, ya que clínicamente nos parece que no cambiaría la conducta clínica luego al indicar terapia posicional. El SAHOS postural fue significativamente más frecuente en los pacientes con enfermedad leve $(75 \%)$, que en las moderadas $(62 \%)$ y las severas $(34 \%)$, prevalencias mayores a la descritas previamente ${ }^{14}$ que correspondían con estudios realizados con PSG y con otra técnica de monitorización de la posición. El porcentaje de sujetos que pudimos analizar adecuadamente, ya que presentaban un registro suficiente en ambas posiciones, fue de un $86 \%$ (503/585). A diferencia del estudio realizado con PSG en la clínica por nuestro grupo, donde un tercio de los pacientes (32\%) no fueron elegibles ya que no presentaban registro suficiente en decúbito lateral para definir si presentaba o no apneas posturales ${ }^{26}$. Esto presenta implicancias diagnósticas ya que al menos en un tercio de los pacientes que se les practica una PSG no vamos a poder responder adecuadamente a esta duda diagnóstica. De igual forma, cuando se realizan estudios de PSG noche dividida es infrecuente observar un tiempo suficiente sueño en ambas posiciones $^{14,27}$, siendo de esta forma muy difícil de evaluar el componente postural en la mayoría de los casos. Esto probablemente se deba a las condiciones en que es realizado los estudios de PSG y al corto tiempo de evaluación en los estudios de noche dividida.

Cuando se compararon los pacientes con SAHOS postural y no postural, los pacientes con AP presentan enfermedad menos severa, menor índice de masa corporal, circunferencia cervical (CC) y frecuencia de hipertensión arterial y son preferentemente mujeres. El estudio clásico de Mador $^{14}$ donde se evaluaron 574 pacientes, las variables que diferenciaban a los pacientes con AP fueron el índice de eventos respiratorios, el IMC y en menor grado los sujetos más jóvenes. La edad no fue un marcador predictivo significativo en nuestro estudio. La utilidad en la práctica clínica habitual de medir rutinariamente la circunferencia cervical queda demostrada ya que es un factor relevante en la relación a la presencia de las apneas posturales, incluso encontramos diferencias entre el grupo de pacientes con AP estricta y no estricta, pudiendo ser un continuo en la fisiopatología de la enfermedad desde el paciente roncador, hipoapnea, AP estricta, AP no estricta 
y AP no P. Los factores más importantes para predecir el SAHOS postural es el índice de eventos respiratorios y la obesidad. El peso evaluado como el IMC, es un factor relevante, de hecho el peso modula el efecto que podría tener las apneas posturales. Se han reportado pacientes que bajando de peso se convirtieron de no posturales a posturales, de igual forma pacientes con SAHOS postural que suben de peso se transforman en no posturales con un aumento significativo del número de eventos respiratorios principalmente en decúbito lateral ${ }^{28}$. No encontramos en el presente estudio diferencias en la somnolencia medida con la escala de Epworth, como tampoco en escala de Stanford, a pesar que en un estudio previo de nuestro grupo los pacientes con SAHOS no postural presentaban mayor somnolencia $(11,3 \pm 0,8$ versus $13,5 \pm 0,5 \mathrm{p}=0,005)$ y una arquitectura del sueño más alterada (mayor sueño etapa I en desmedro del sueño profundo y REM, asociado a aumento de los micro despertares) ${ }^{26}$.

El análisis multivariado confirmó la menor probabilidad de presentar SAHOS postural en los sujetos con SAHOS severo (OR 0,96: IC 0,94$0,97)$ : los hipertensos $(0,54$ : IC $0,33-0,88)$ y en los hombres (OR 0,41, IC 0,21-0,81).

La importancia de conocer y diagnosticar al grupo de pacientes con SAHOS postural es que podría verse beneficiado de medidas posicionales a través de una variedad de dispositivos descritos en la literatura ${ }^{29}$, por lo tanto, debe investigarse su presencia. La eficacia de algunas de estas medidas ha sido evaluada y estos dispositivos son capaces de disminuir la cantidad de sueño en posición supina y como resultado de esto disminuir la severidad del SAHOS. Una revisión sistemática reciente ${ }^{20}$ encontró sólo tres estudios de calidad satisfactoria para evaluar SAHOS postural comparándola con $\mathrm{CPAP}^{30-32}$, de esta se concluye que la CPAP fue más efectiva en reducir el índice de eventos respiratorios y aumentar la saturación de oxígeno, aunque estas diferencias fueron pequeñas y clínicamente no significativas, la ventaja del CPAP fue aún menor en los pacientes con apnea leve, considerando que la aceptación y tolerancia al CPAP es muchas veces problemática en estos pacientes ${ }^{33}$, particularmente en ausencia de somnolencia diurna, donde la opción de terapia postural cobra mayor relevancia.

En conclusión, el SAHOS postural definido como la reducción del 50\% o más en el índice de eventos respiratorios de la posición supina a la lateral es una entidad muy frecuente y se presenta en el $53 \%$ de los pacientes con SAHOS, aun utilizando un criterio más estricto de AP (IAH < 10 eventos/h en decúbito lateral) esta condición sigue siendo muy frecuente siendo identificada en un tercio de los pacientes con SAHOS. Los pacientes con SAHOS postural presentan una enfermedad menos severa, un índice de masa corporal y circunferencia cervical menor, menor frecuencia de hipertensión arterial y son preferentemente mujeres. Creemos que la definición más estricta de AP es la más adecuada ya que presenta connotaciones terapéuticas relevantes.

\section{Bibliografía}

1.- YOUNG T, PALTA M, DEMPSEY J, SKATRUD J, WEBER S, BADR S. The occurrence of Sleep disorders breathing among middle aged adults. N Engl J Med 1993; 328: 1230-5.

2.- DURÁN J, ESNAOLA S, RAMÓN R, IZTUETA A. Obstructive sleep apnea-hypopnea and related clinical features in a population-based sample of subjects aged 30 to 70 years. Am J Respir Crit Care Med 2001; 163: 685-9.

3.- BALDWING. BALDWIN CM, GRIFFITH KA, NIETO FJ, O'CONNOR GT, WALSLEBEN JA, REDLINE S. The association of sleep-disordered breathing and sleep symptoms with quality of life in the Sleep Heart Healt Study. Sleep 2001; 24: 96-105.

4.- PEPPARD P E, YOUNG T, PALTA M, SKATRUD J. Prospective study of the association between sleepdisordered breathing and hypertension. New Eng J Med 2000; 342: 1378-84.

5.- DURÁN-CANTOLLA J, AIZPURU F, MARTÍNEZNULL C, BARBÉ ILLA F. Obstructive sleep apnea/ hypopnea and systemic hypertension. Sleep Med Rev 2009; 13: 323-31.

6.- CANO-PUMAREGA I, DURÁN-CANTOLLA J, AIZPURU F, MIRANDA-SERRANO E, RUBIO R, MARTÍNEZ-NULL C, et al. On behalf of the Spanish Sleep and Breathing Group. Obstructive sleep apnea and systemic hypertension: Longitudinal study in the general population: The Vitoria Sleep Cohort. American Journal Respir Critical Care Med 2011; 184: 1299-304.

7.- MARÍN J M, AGUSTI A, VILLAR I, FORNER M, NIETO D, CARRIZO S J, et al. Association between treated and untreated obstructive sleep apnea and risk of hypertension. JAMA 2012; 307: 2169-76.

8.- YOUNG T, BLUSTEIN J, FINN L, PALTA M. Sleepdisordered breathing and motor vehicle accidents in a population-based sample of employed adults. Sleep 1997; 20: 608-13.

9.- TERÁN-SANTOS J, JIMÉNEZ-GÓMEZ A, CORDERO-GUEVARA J, AND THE COOPERATIVE GROUP BURGOS-SANTANDER. The association between sleep apnea and the risk of traffic accidents. N Engl J Med 1999; 340: 847-51.

10.- MARSHALL N S, WONG K K H, LIU P Y, CULLEN 
S R J, KNUIMAN M W, GRUNSTEIN R R. Sleep apnea as an independient risk factor for all-cause mortality, The Busselton Health Study. Sleep 2008; 31; 1079-85.

11.- MARÍN J M, CARRIZO S J, VICENTE E, AGUSTÍ A $\mathrm{G} N$. Long-term cardiovascular outcomes in men with obstructive sleep apnoea-hypopnoea with or without treatment with continuous postiive airway pressure: an observational study. Lancet 2005; 365: 1046-53.

12.- YOUNG T, FINN L, PEPPARD P, SZKLO-COXE M, AUSTIN D, NIETO J, STUBBS R, HLA K M. Sleepdisordered-Breathing and mortality; Eigtheen-year follow-up of Wisconsin Sleep Cohort. Sleep 2008; 31 : 1071-8.

13.- OKSENBERG A, SILVERBERG D S, ARONS E, RADWAN H. Positional vs nonpositional obstructive sleep apnoea patients. Anthropomorphic, nocturnal polysomnographic and multiple sleep latency test data. Chest 1997; 112: 629-39.

14.- MADOR M J, KUFEL T J, MAGALANG U J, RAJESH S K, WATWE V, GRANT J B. Prevalence of positional sleep apnoea in patients undergoing polysomnography. Chest 2005; 128: 2130-7.

15.- RICHARD W, KOX D, DEN HERDER C, LAMAN M, VAN TINTEREN H, DE VRIES N. The role of sleep position in obstructive sleep apnoea syndrome. Eur. Arch. Otorhinolaryngol 2006; 263: 946-50.

16.- OKSENBERG A, ARONS E, GREENBERG-DOTAN $\mathrm{S}$, NASSER K, RADWAN H. The significance of body posture on breathing abnormalities during sleep: data analysis of 2077 obstructive sleep apnoea patients. Harefuah 2009; 148: 157-62.

17.- DURÁN-CANTOLLA J T L, PUERTAS-CUESTA F J, PIN-ARBOLEDAS G; GRUPO ESPAÑOL DE SUEÑO (GES). Documento de consenso nacional sobre el síndrome de apneas hipopneas del sueño. Arch Bronconumol 2005; 41: 1-110.

18.- LLOBERES P, DURÁN-CANTOLLA J, MARTÍNEZGARCÍA M A, MARÍN J M, FERRER A, CORRAL J, et al. Diagnóstico y tratamiento del síndrome de apneashipopneas del sueño. Arch Bronconeumol 2011; 47 : 143-56.

19.- GILES T L, LASSERSON T J, SMITH B J, WHITE J, WRIGHT J, CATES C J. Continuous positive airways pressure for obstructive sleep apnea in adults. Cochrane Database Syst Rev 2006; (1): CD001106.

20.- HA S C, HIRAI H W, TSOI K K. Comparison of positional therapy versus continuous positive airway pressure in patients with positional obstructive sleep apnea: a meta-analysis of randomized trials. Sleep Med Rev 2014; 18: 19-24.

21.- OSMAN E Z, OSBORNE J, HILL P D, LEE B W. The Epworth Sleepiness Scale: Can it be used for sleep apnoea screening among snorers? Clin Otolaryngol Allied Sci 1999; 24: 239-41.

22.- VANDEPUTTE M, DE WEERD A. Sleep disorders and depressive feelings: a global survey with the Beck depression scale. Sleep Med 2003; 4: 343-5.

23.- FERBER R, MILLMAN R, COPPOLA M, FLEETHAM J, MURRAY CF, IBER C, et al. Portable recording in the assessment of obstructive sleep apnea. Sleep 1994; 17: 378-92.

24.- IBER C, ANCOLI-ISRAEL S, CHESSON A, QUAN S. For the American Academy of Sleep Medicine. The AASM Manual for the Scoring of Sleep and Associated Events: Rules, Terminology and Technical Specifications. 2007. 1st edition. Westchester, IL: American Academy of Sleep Medicine.

25.- MCNICHOLAS W T. Diagnosis of obstructive sleep apnea in adults. Proc Am Thorac Soc 2008; 5: 154-60.

26.- JORQUERA J, FREIRE M, SANTÍN J, GODOY J. Apnea obstructiva del sueño con componente postural; diferencias clínicas y polisomnográficas. Rev Chil Enferm Respir 2002; 18: 286 Resumen P28.

27.- JORQUERA J, SANTÍN J, GODOY J. La polisomnografía en noche dividida es eficaz para titular la presión en la ventilación con presión positiva continua (“CPAP”) en pacientes con apnea obstructiva del sueño. Rev Med Chile 134: 1377-82.

28.- OKSENBERG A, DYNIA A, NASSER K. GADOTH N. Obstructive sleep apnoea in adults: body postures and weight changes interactions. J Sleep Res 2012; 21 : 402-9.

29.- RAVESLOOT M J L, VAN MAANEN J P, DUN L, DE VRIES N. The undervalued potential of positional therapy in position-dependent snoring and obstructive sleep apnea-a review of the literature. Sleep Breath 2013; 17: 39-49.

30.- PERMUT I, DÍAZ-ABAD M, CHATILA W, CROCETTI J, GAUGHAN J P, D’ALONZO G E, et al. Comparison of positional therapy to CPAP in patients with positional obstructive sleep apnea. J Clin Sleep Med 2010; 6: 238-43.

31.- SKINNER M A, KINGSHOTT R N, FILSELL S, TAYLOR D R. Efficacy of the "tennis ball technique" versus nCPAP in the management of position-dependent obstructive sleep apnoea syndrome. Respirology 2008; 13: 708-13.

32.- JOKIC R, KLIMASZEWSKI A, CROSSLEY M, SRIDHAR G, FITZPATRICK M F. Positional treatment vs continuous positive airway pressure in patients with positional obstructive sleep apnea syndrome. Chest 1999; 115: 771-81.

33.- SANTIN J, JORQUERA J, JORDAN J, GODOY J. Uso de CPAP nasal en el largo plazo en síndrome de apnea hipopnea del sueño. Rev Med Chile 2007; 135: 855-61.

Correspondencia a:

Dr. Jorge Jorquera Arévalo

Enfermedades Respiratorias Clínica Las Condes.

Lo Fontecilla 441, edificio azul $2^{\circ}$ piso. Las Condes.

Email: jjorquera@clc.cl 